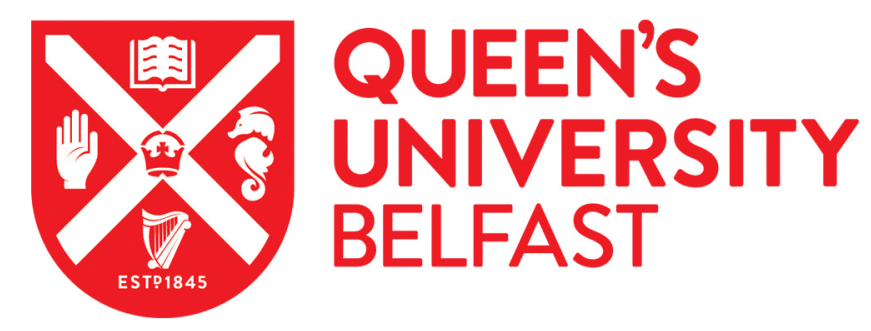

\title{
Generation of a Purely Electrostatic Collisionless Shock during the Expansion of a Dense Plasma through a Rarefied Medium
}

Sarri, G., Dieckmann, M. E., Kourakis, I., \& Borghesi, M. (2011). Generation of a Purely Electrostatic Collisionless Shock during the Expansion of a Dense Plasma through a Rarefied Medium. Physical Review Letters, 107(2), [025003]. https://doi.org/10.1103/PhysRevLett.107.025003

Published in:

Physical Review Letters

Document Version:

Publisher's PDF, also known as Version of record

Queen's University Belfast - Research Portal:

Link to publication record in Queen's University Belfast Research Portal

Publisher rights

(c) 2011 American Physical Society

\section{General rights}

Copyright for the publications made accessible via the Queen's University Belfast Research Portal is retained by the author(s) and / or other copyright owners and it is a condition of accessing these publications that users recognise and abide by the legal requirements associated with these rights.

Take down policy

The Research Portal is Queen's institutional repository that provides access to Queen's research output. Every effort has been made to ensure that content in the Research Portal does not infringe any person's rights, or applicable UK laws. If you discover content in the Research Portal that you believe breaches copyright or violates any law, please contact openaccess@qub.ac.uk. 


\title{
Generation of a Purely Electrostatic Collisionless Shock during the Expansion of a Dense Plasma through a Rarefied Medium
}

\author{
G. Sarri, ${ }^{1}$ M. E. Dieckmann, ${ }^{2}$ I. Kourakis, ${ }^{1}$ and M. Borghesi ${ }^{1}$ \\ ${ }^{1}$ Centre for Plasma Physics, The Queens University of Belfast, Belfast BT7 1NN, United Kingdom \\ ${ }^{2}$ Department of Science and Technology (ITN), Linköping University, 60174, Norrkoping, Sweden
}

(Received 29 April 2011; published 6 July 2011)

\begin{abstract}
A two-dimensional numerical study of the expansion of a dense plasma through a more rarefied one is reported. The electrostatic ion-acoustic shock, which is generated during the expansion, accelerates the electrons of the rarefied plasma inducing a superthermal population which reduces electron thermal anisotropy. The Weibel instability is therefore not triggered and no self-generated magnetic fields are observed, in contrast with published theoretical results dealing with plasma expansion into vacuum. The shock front develops a filamentary structure which is interpreted as the consequence of the electrostatic ion-ion instability, consistently with published analytical models and experimental results.
\end{abstract}

The collision of plasma clouds with different properties (such as temperature, density, and composition) is a fairly common phenomenon in nature [1]. The high degree of nonlinearity of such collisions facilitates the onset of collisionless shock waves (CSWs) [2,3] that, in the case of unmagnetized plasmas, can be of two different kinds: either electrostatic or Weibel-induced. For instance, a supernova explosion [4] accelerates, in a plasma state, a significant fraction of the stellar mass up to a few percent of the speed of light [5]. The propagation of this plasma through the more tenuous interstellar medium induces the excitation of CSWs which have been recently suggested to play a fundamental role in the generation of highly energetic particle bunches and cosmic $\gamma$ rays [6]. In this case, the high currents associated with these energetic particles induce such CSWs to be strongly magnetized [7]. However, purely electrostatic, nonrelativistic CSWs might be present in other astrophysical scenarios of great interest such as microquasar systems [8]. In these binary systems, the outflow of matter from the rotating star to the accreting black hole takes initially the form of a transient, highly relativistic jet which is followed by a slower, radioemitting, accretion disk [9]. These systems are of central relevance to understanding the dynamics involved in higher scale, ultramassive binary systems, whose slow dynamics rules out a fully characterized observation. Purely electrostatic, nonrelativistic CSWs can also be generated around ultramassive stars (such as the Herbig $\mathrm{Ae} / \mathrm{Be}$ stars [10]) whose atmosphere has been demonstrated to be completely unmagnetized [11].

CSWs can nowadays be recreated also in laboratory experiments comprising the interaction of a high energy laser pulse with a solid target. In this case CSWs can be generated during the expansion of the laser-ablated plasma through the more tenuous plasma resulting from the photoionization of the residual gas embedding the solid target $[12,13]$. This particular scenario has been proven to be of direct relevance to a wide range of possible applications which include laser-driven particle acceleration [14,15] and inertial confinement fusion [16].

From the theoretical point of view, plasma expansion has been studied, both analytically and numerically, mostly in the assumption of expansion into vacuum [17-20]. In this case, the electrons of the expanding plasma lose kinetic energy exclusively along the expansion direction [20]. The subsequent anisotropic thermal energy distribution triggers the onset of the Weibel instability [21-23] inducing a magnetized plasma front [20]. Filamented fronts have been numerically observed and interpreted to be a consequence of this instability $[20,24]$. Plasma expansion and CSWs in a purely electrostatic regime (as the ones reported in Refs. $[12,13]$ ) cannot be explained within this framework.

In this Letter, we give the first numerical demonstration that the presence of a rarefied ambient plasma can forbid magnetic field generation by acting as a suppressor for the Weibel instability at the plasma front, leading to a purely electrostatic evolution of the plasma. The electrons of the ambient plasma are in fact accelerated by a CSW triggered ahead of the expanding plasma, and form a superthermal population which significantly reduces the anisotropy in the electron temperature [25]. Our simulation shows in fact a very modest cooling of the electrons along the plasma propagation direction. The CSW is still seen to develop a filamented front, as experimentally detected in Ref. [13]. However, these filaments are interpreted to be triggered not by the Weibel instability but by the electrostatic ion-ion instability [26,27]. This instability has been found to be of relevance not only to purely electrostatic fronts but also in partially magnetized fronts (such as the foot of magnetized CSWs in supernova remnants [28]).

We simulated the expansion of a dense plasma into a more rarefied one, via a two-dimensional particle-in-cell code [29]. This work follows from an earlier study, restricted to one spatial dimension, whose discussion can 
be found in [30]. The simulation box dimension is $L_{x} \times$ $L_{y}=540 \lambda_{D} \times 60 \lambda_{D}$ with $\lambda_{D}$ the Debye length of the rarefied plasma. The box is divided along $x$ into two halves. The interval $-L_{x} / 2<x<0$ contains a plasma with density $n_{0}=10^{17} \mathrm{~cm}^{-3}$ whereas the interval $0<x<L_{x} / 2$ contains a plasma with density $n_{0} / 100$. Both plasmas have an electron (proton) temperature of $1 \mathrm{keV}(10 \mathrm{eV})$. Temperatures and densities have been chosen in order to be comparable with the experimental conditions reported in Ref. [13] and of relevance to the accretion disks in microquasar systems (measured blackbody radiation consistent with the presence of $\mathrm{keV}$ electrons [9]). The full simulation domain is resolved by a grid with $4000 \times 440$ cells and all boundary conditions are periodic. 360 (160) computational particles per simulation cell are used to represent the dense (rarefied) plasma species. The simulation ran for approximately half a nanosecond.

The sharp density jump located at $x=0$ induces the electrons of the higher density plasma to immediately diffuse; the heavier protons are not able to keep up with this motion and a net charge imbalance is set close to the plasmas' interface. The resulting positive electrostatic field [Fig. 1(c)] accelerates the protons of both plasmas leading to a bending in the proton phase space [Fig. 1(a)] which evolves into a shocklike structure that interrupts the rarefaction wave, approximately at $x=0.2 \mathrm{~mm}$ for $t_{2}=$ $0.35 \mathrm{~ns}$ [Fig. 1(b)]. This structure presents a monopolar electrostatic field [Fig. 1(d)] and propagates with a velocity of the order of the ion-acoustic speed of the tenuous plasma. An analogous structure is not observed in the electron phase space [compare Figs. 1(b) and 3(a)], corroborating the thesis of an ionic CSW.

The most interesting result arising from direct analysis of the simulation output is that no significant magnetic fields are generated throughout the entire simulation box (Fig. 2). The maximum fluctuation of the mean values of the magnetic $B_{y}$ and $B_{z}$ components are kept extremely low across the entire simulation box $\left[\left\langle B_{y}\right\rangle_{y},\left\langle B_{z}\right\rangle_{y} \leq 5 \times\right.$ $10^{-3} \mathrm{~T}$, see Figs. 2(a) and 2(b)]. This deviation from zero follows from the finite number of cells $\left(N_{y}=440\right)$ over
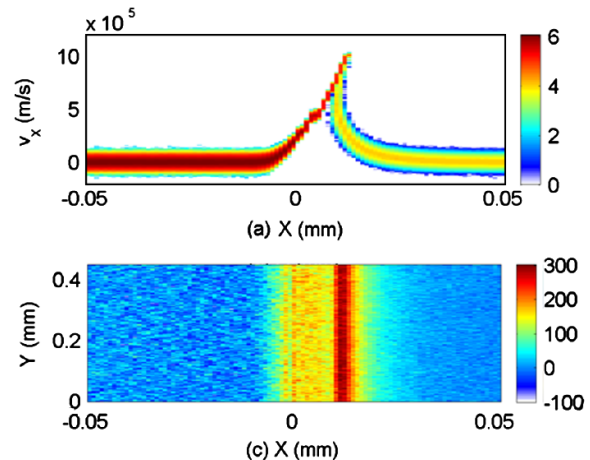

which the averaging is performed. However, such mean values are much smaller than the related standard deviation $[\approx 0.2 \mathrm{~T}$, see Fig. 2(c)]. This, together with a standard deviation which is practically constant along $z$, implies that there is no underlying signal in the statistical noise induced by the particle-in-cell representation of the plasma. The standard deviation of $B_{y}$ (not shown) followed a similar trend. The plasma dynamics, as well as the CSW generated, are therefore of purely electrostatic nature. This result is in contrast to simulations of plasma expansion into vacuum reported in literature (see, for instance, Ref. [20]). During expansion into vacuum, the electrons of the plasma lose kinetic energy exclusively along the expansion axis, due to the transfer of energy from the electrons to the expanding protons. The electron velocity distribution develops thus an anisotropy which feeds the Weibel instability inducing a magnetized plasma front. A nonthermal (cold) electron population in the expanding plasma does not effectively change the plasma dynamics [20], suggesting that magnetic fields are always created in this scenario, regardless of the electron distribution function of the expanding plasma. However, if the plasma is forced to expand in a tenuous medium, a population of electrons is present ahead of the plasma front. Such a population is accelerated by the CSW and develops a superthermal distribution. These electrons reduce the gap between the thermal energy along the transverse and longitudinal directions and therefore limit the thermal anisotropy to be well below $10 \%$ for the entire duration of the simulation [see Fig. 3(b)]. This anisotropy is not sufficient to trigger significant magnetic fields (Fig. 2). The dispersion relation for the Weibel instability can in fact be expressed, in its linear phase, as [23]

$$
\frac{k_{w}^{2} c^{2}}{\omega_{p e}^{2}}+\frac{\sigma^{2}}{\omega_{p e}^{2}}=-\left[1+\frac{1}{2}(A+1) Z^{\prime}\left(\frac{i \sigma}{k_{w} v_{t h}}\right)\right]
$$

where $Z$ is the plasma dispersion function [31]:

$$
Z(s)=e^{-s^{2}}\left(i \pi^{1 / 2}-2 \int_{0}^{s} d t e^{t^{2}}\right)
$$
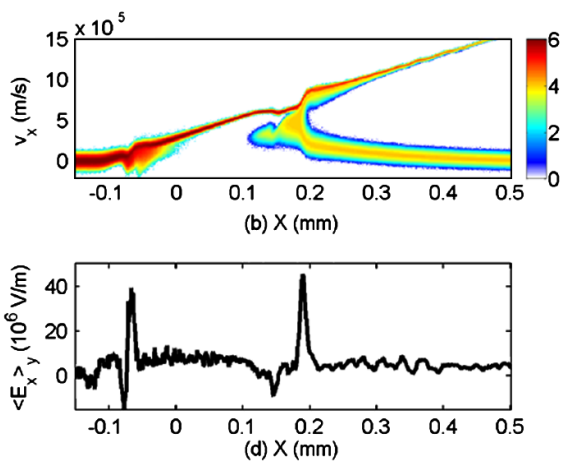

FIG. 1 (color online). Proton phase space distributions at $t_{1}=15 \mathrm{ps}$ (a) and $t_{2}=0.35 \mathrm{~ns}$ (b). The color scale is 10 -logarithmic and in units of the average density of the tenuous plasma. (c) Spatial distribution of the electric field amplitude at $t_{1}=15$ ps. (d) Electric field distribution $E_{x}$, averaged over $y$ for $t_{2}=0.35 \mathrm{~ns}$. Electric fields are in unit of $10^{6} \mathrm{~V} / \mathrm{m}$. 

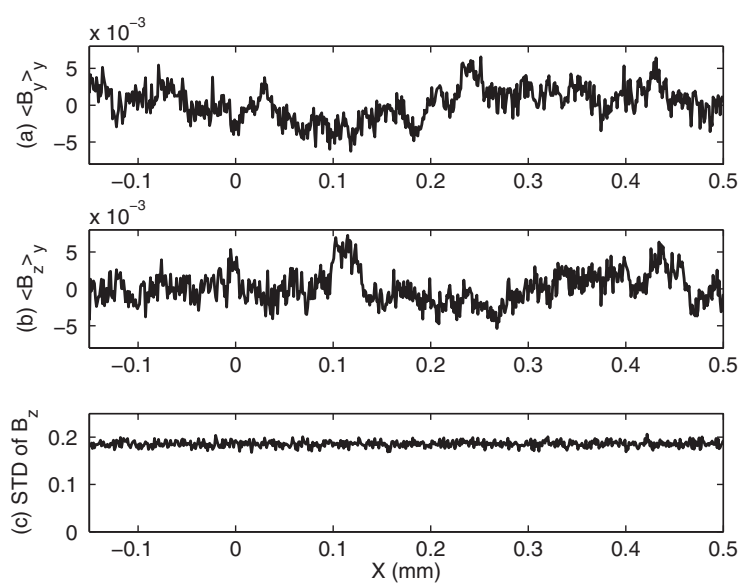

FIG. 2. Mean values of $B_{y}$ (a) and $B_{z}$ (b), averaged along the $y$ direction, and the standard deviation of $B_{z}$ along the $y$ direction (c). All graphs refer to $t_{2}=0.35 \mathrm{~ns}$ and are in units of Tesla.

Here $A=\left(v_{\perp} / v_{\|}\right)^{2}-1$ indicates the degree of anisotropy of the electron thermal velocities, $k_{w}$ and $\sigma$ are the wave number and the linear growth rate $(\omega=i \sigma)$ associated with the instability. Following the definition of $\sigma$, the instability is triggered only if $\sigma^{2}>0$. Using Fig. 3(b), $A$ can be readily estimated to be $A \leq 0.1$. For this value, Eq. (1) indicates that $\sigma^{2}>0$ only for $k_{w}<6 \times 10^{3} \mathrm{~m}^{-1}$ or, equivalently, $\lambda_{w}>1 \mathrm{~mm}$. The presence of the CSW reduces the extension of the rarefaction wave to much less than $1 \mathrm{~mm}$ [see Fig. 1(b)] thus forbidding the Weibel instability to be triggered. On the other hand, for plasmas expanding into vacuum $A$ can be of the order of few tens $[20,23]$. In this regime Eq. (1) implies purely growing modes maximized for a wavelength of the order of the electron Debye length of the ambient plasma.

These considerations are applicable also to laser-heated plasmas in which the electrons are initially anisotropically
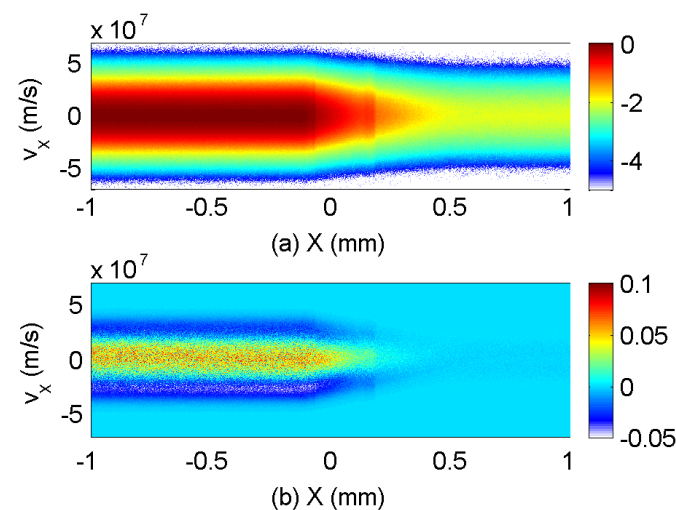

FIG. 3 (color online). (a) Electron phase space at $t_{2}=0.35 \mathrm{~ns}$. The color scale is 10-logarithmic and in units of the average density of the dense plasma. (b) Difference between the electron distribution function at $t_{2}=0.35 \mathrm{~ns}$ and the initial Maxwellian distribution. The color scale is normalized to the peak of the distribution. heated by the laser pulse leading to a temperature along the longitudinal dimension that is larger than along the transverse one [32]. This anisotropy is in fact smoothed out by the cold electrons in the expanding plasma in a time scale of few femtoseconds [20,33]. In the case of plasma expansion into vacuum, persistent Weibel instability is triggered only at later times, when the longitudinal momentum of the electrons falls below the transverse one, due to the cooling induced by energy transfer from the electrons to the expanding protons [20].

Despite the absence of magnetic fields, a filamentary structure of the shock front is still visible in our simulation results as shown in Fig. 4(a). Such filaments are associated with a relative proton density modulation of around $10 \%$ and a spatial period of the order of few times the Debye length of the tenuous plasma. They appear to be mostly transverse to the shock front, showing an average angular deviation from the front's perpendicular of about $10^{\circ}$. Such modulations are also present in the electron density map (not shown) and are here induced by the conservation of charge neutrality. However, the much higher thermal speed of the electrons implies that this density modulation is much weaker (less than 1/1000) and can therefore not fully compensate that of the protons inducing a net electrostatic field. This field has a maximum amplitude of $5 \times 10^{7} \mathrm{~V} / \mathrm{m}$ and it is predominantly along the $y$ direction [Fig. 4(c)], just ahead of the shock front [Fig. 4(d)]. The $x$ component of the electrostatic field shows a much weaker modulation $\left(\approx 10^{6} \mathrm{~V} / \mathrm{m}\right)$ together with a localized signal associated with the shock itself [Fig. 4(b)]. At first sight, such filaments appear to be similar to the ones shown in Ref. [24] which have been interpreted to be a consequence of the Weibel instability. However, the lack of magnetic fields discussed above gives a clear evidence of the different nature of the filaments detected in our simulation. We interpret these filaments as being triggered by the ion-ion instability [26,27]. This instability can occur when an ion beam traverses an electron-ion plasma only if
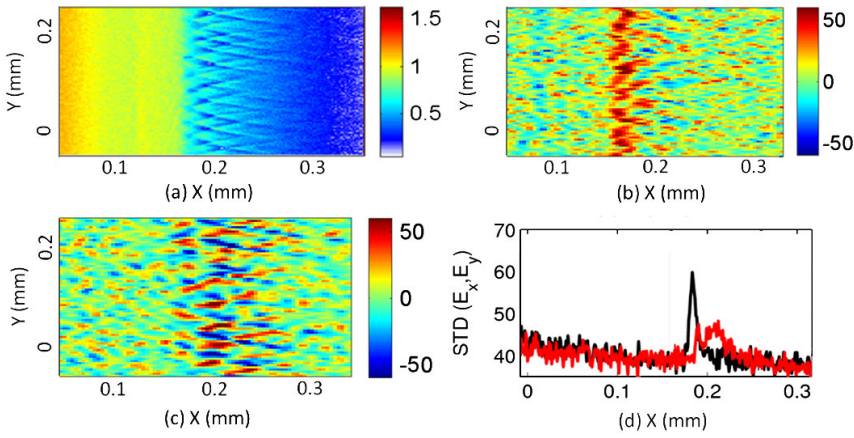

FIG. 4 (color online). (a) Proton density map, integrated over $v_{x}$, in units of the initial density of the dense plasma. The related electric field components $E_{x}$ and $E_{y}$ are displayed in (b) and (c), respectively. The color scale is in units of MV. The standard deviation of $E_{x}$ (black) and $E_{y}$ [gray (red)], in units of $10^{6} \mathrm{~V} / \mathrm{m}$, is shown in $(d)$. 
the electron to ion temperature ratio is high enough to avoid Landau damping of ion-acoustic waves. In our simulation, the proton beam is provided by the shock-reflected ions (whose velocity is thus equal to 2 times the ionacoustic speed of the tenuous plasma) and the ratio between the electron and ion temperature is 100, large enough to allow the onset of the instability, as numerically shown in Ref. [27]. From an analytical point of view, the Debye wave number of the ambient plasma ions $\left(k_{i}\right)$ and that of the instability $\left(k_{s}\right)$ are related by [26]

$$
2 \frac{k_{s}^{2}}{k_{i}^{2}}=Z^{\prime}(u)+n \alpha^{2} Z^{\prime}[\alpha(u-V)]-2(n+1) T .
$$

Here $Z$ is the plasma dispersion function as defined in Eq. (2), $n$ is the ratio between the ion density of the tenuous plasma and the propagating beam, $T=T_{i} / T_{e} \approx 1 / 100, \alpha$ is the thermal speed of the ion beam, $u$ is the phase speed, and $V$ is the drift speed of the ion beam. All velocities are normalized in terms of the ion thermal speed of the tenuous plasma $v_{\text {thi }}$. For our simulation parameters $\alpha \approx 1, n$ is of the order of unity, and $V \approx 2 c_{s} / v_{\text {thi }} \approx 20$. In this regime the damping component of Eq. (2) is negligible, as also confirmed by comparing our parameters with Ref. [27]. Equation (3) gives therefore $k_{s}^{2} / k_{i}^{2} \approx 0.04$, which implies that the filaments have a spatial period of the order of 5 times the ion Debye length of the ambient plasma $\lambda_{D \text { amb }}$. The average ion density ahead of the shock is approximately 20 times the initial ion density of the rarefied plasma [see Fig. 4(a)] implying $\lambda_{D \mathrm{amb}} \approx 4 \lambda_{D i}$. Considering that, for our simulation parameters, $\lambda_{D i}=$ $\lambda_{D e} / 10$, the spatial period of the filaments is calculated to be of the order of 2 times the electron Debye length of the rarefied plasma (i.e., $\approx 15 \mu \mathrm{m}$ ), in good agreement with our simulation findings. This result is also in good agreement with the theoretical results reported in Ref. [27].

It is interesting to note that our simulation results are consistent with the filamented shock front experimentally observed in Ref. [13]. In this Letter, a modulation in the probing proton beam is detected in the foreshock region (see region III in Fig. 1 of Ref. [13]) generated during the expansion of a dense plasma, resulting from laser-solid ablation, through a more rarefied one. The striations detected were purely electrostatic, with a typical width of the order of the Debye length of the rarefied plasma and appear to be almost perpendicular to the shock fronts, all elements in agreement with our simulation results.

In conclusion, we have carried out a two-dimensional particle-in-cell simulation aimed at studying the propagation of a dense plasma into a more rarefied one. The expansion of the dense plasma launches an ion-acoustic collisionless shock that accelerates the electrons of the rarefied plasma. These electrons significantly reduce the anisotropy in the electron temperature, brought about by the energy transfer to the protons along the expansion direction. The Weibel instability is therefore hampered and no magnetic fields are generated for the entire duration of the simulation. The shock develops a filamented front which has been interpreted as a consequence of the ion-ion instability. These results significantly differ from analytical and numerical treatments of plasma expansion into vacuum and are of relevance to understanding both astrophysical plasma scenarios and laser-plasma experiments performed in similar conditions.

This work has been supported by the UK Engineering and Physical Sciences Research Council (Grant No. EP/ D06337X/1), GK 1203, and Vetenskapsrådet Sweden (Grant No. 2010-4063).

[1] T.K. Gaisser, Cosmic Rays and Particle Physics (Cambridge University Press, Cambridge, England, 1990).

[2] T. M. O’Neil and F. V. Coroniti, Rev. Mod. Phys. 71, S404 (1999).

[3] B. Eliasson and P. K. Shukla, Phys. Rep. 422, 225 (2006).

[4] K. Koyama et al., Nature (London) 378, 255 (1995).

[5] S. R. Kulkarni et al., Nature (London) 395, 663 (1998).

[6] Y. Uchiyama et al., Nature (London) 449, 576 (2007).

[7] A. R. Bell and S. G. Lucek, Mon. Not. R. Astron. Soc. 321, 433 (2001).

[8] I. F. Mirabel et al., Nature (London) 358, 215 (1992).

[9] R. Fender and T. Belloni, Astron. Astrophys. 42, 317 (2004).

[10] G. H. Herbig, Astrophys. J. Suppl. Ser. 4, 337 (1960).

[11] J. S. Vink et al., Mon. Not. R. Astron. Soc. 337, 356 (2002).

[12] Y. Kuramitsu et al., Phys. Rev. Lett. 106, 175002 (2011).

[13] L. Romagnani et al., Phys. Rev. Lett. 101, 025004 (2008).

[14] S. Hatchett et al., Phys. Plasmas 7, 2076 (2000).

[15] P. Mora, Phys. Rev. Lett. 90, 185002 (2003).

[16] M. Tabak et al., Phys. Plasmas 1, 1626 (1994).

[17] C. Sack and H. Schamel, Phys. Rep. 156, 311 (1987).

[18] P. Mora, Phys. Rev. E 72, 056401 (2005).

[19] P. Mora and T. Grismayer, Phys. Rev. Lett. 102, 145001 (2009).

[20] C. Thaury, P. Mora, A. Héron, J. C. Adam, and T. M. Antonsen, Phys. Rev. E 82, 026408 (2010).

[21] E. S. Weibel, Phys. Rev. Lett. 2, 83 (1959).

[22] L. Palodhi, F. Califano, and F. Pegoraro, Plasma Phys. Controlled Fusion 51, 125006 (2009).

[23] A. Stockem, M.E. Dieckmann, and R. Schlickeiser, Plasma Phys. Controlled Fusion 52, 085009 (2010).

[24] T. N. Kato and H. Takabe, Phys. Plasmas 17, 032114 (2010).

[25] M. Lazar, R. Schlickeiser, and S. Poedts, Phys. Plasmas 17, 062112 (2010).

[26] B. D. Fried and A. Y. Wong, Phys. Fluids 9, 1084 (1966).

[27] S. P. Gary and N. Omidi, J. Plasma Phys. 37, 45 (1987).

[28] Y. Ohira and F. Takahara, Astrophys. J. 688, 320 (2008).

[29] J. M. Dawson, Rev. Mod. Phys. 55, 403 (1983).

[30] G. Sarri, M. E. Dieckmann, I. Kourakis, and M. Borghesi, Phys. Plasmas 17, 082305 (2010).

[31] B.D. Fried and S.D. Conte, The Plasma Dispersion Function (Academic, New York, 1961).

[32] F. N. Beg et al., Phys. Plasmas 4, 447 (1997).

[33] C. Thaury, P. Mora, A. Héron, and J. C. Adam, Phys. Rev. E 82, 016408 (2010). 